\title{
QUALIDADE DE VIDA DE PRATICANTES DE DANÇA EM ACADEMIAS NO PERÍODO PANDÊMICO DA COVID-19
}

\section{QUALITY OF LIFE OF DANCE STUDENTS IN GYMS DURING THE COVID-19 PANDEMIC}

\author{
Brenda de Carvalho Silva Brito', Gabriel Rodrigues Neto", Júlio César Gomes da SilvalII*
}

\begin{abstract}
Resumo. Mesmo com os benefícios, trazidos pela dança, em diversos aspectos relacionados à saúde e ao condicionamento físico de indivíduos, de diferentes faixas etárias, percebe-se que poucos estudos na literatura investigam a qualidade de vida de praticantes de dança em academias. Desta forma, o objetivo do presente estudo é analisar a percepção da qualidade de vida de praticantes de dança em academias na cidade de João Pessoa-PB no período pandêmico da COVID-19. A amostra foi composta por 101 pessoas, entre homens e mulheres, praticantes de dança em academias da cidade de João Pessoa. A percepção da qualidade de vida foi verificada por meio do questionário WHOQOL-bref. Os dados foram analisados no pacote estatístico computadorizado Statistical Package for the social Science, versão 21.0. Inicialmente, foi realizado o teste de distribuição de frequência absoluta e relativa para análise dos dados referentes a prática da dança e aos domínios da qualidade de vida. Os principais achados deste estudo apontam que: a) a maioria dos praticantes de dança em academias é do sexo feminino (85,1\%); b) apresentam uma qualidade de vida satisfatória, como também, uma melhor percepção de qualidade de vida no domínio relações sociais $(77,2 \%)$. Conclui-se que as aulas de dança em academias são compostas, em sua grande maioria, pelo público feminino e, ainda, que a dança em academias, por seus escores satisfatórios, pode ser um instrumento para a melhoria/manutenção da qualidade de vida.
\end{abstract}

PALAVRAS-CHAVE: Saúde Mental. Pandemia. Exercício Físico. Questionários.

Abstract. Despite the benefits of dancing in several aspects related to health and physical conditioning of individuals of different age groups, it is noticeable that few studies in the literature explore the quality of life of dance students in gymnasiums. Thus, the objective of this study is to analyze the perception of the quality of life of dance students in gyms in João Pessoa - PB. The sample was composed by 101 people, among men and women, dance students in gyms in João Pessoa. The WHOQOL-bref Questionnaire was used to analyze the perception of the QL. The data were analyzed in the computerized Statistical Package for the social Science, version 21.0. Initially, the absolute and relative frequency distribution test was performed for the analysis of the data concerning the practice of dance and the domains of quality of life. The main findings of this study show that: a) the majority of dance students in gyms is female (85.1\%); and b) they present a satisfactory quality of life, however, they present a better perception of the quality of life in the domain of social relationships (77.2\%). It is concluded that dance classes in gyms are composed, in their great majority, by th e female audience, finally dance in gyms can be an instrument for the improvement/ maintenance of the quality of life, because there are satisfactory scores for the domains of quality of life.

KEYWORDS: Mental Health. Pandemic. Physical Exercise. Questionnaire.

'Graduada em Bacharelado em Educação Física. Faculdade de Enfermagem Nova Esperança - FACENE. CEP: 58052-310, João Pessoa, Paraíba, Brasil. ORCID ID: 0000-0002-4931-9405.

IIProfissional de Educação Física. Doutor em Educação Física. Professor da Faculdade de Enfermagem Nova Esperança - FACENE, Departamento de Educação Física. CEP: 58052-310, João Pessoa, Paraíba, Brasil. ORCID ID: 0000-0001-8477-1993.

III Profissional de Educação Física. Mestre em Educação Física. Professor da Faculdade de Enfermagem Nova Esperança - FACENE, Departamento de Educação Física. CEP: 58052-310, João Pessoa, Paraíba, Brasil. *Autor correspondente: juliociesar123@gmail.com. ORCID ID: 0000-0002-3128-1883. 


\section{INTRODUÇÃO}

Dentre as atividades realizadas pelo homem, a dança se evidencia como uma expressão viva e presente nas diversas relações humanas. A dança, independentemente de onde, ou como se vive, é considerada um epicentro de transformação social. ${ }^{1,2}$ Neste sentido, é considerada uma forma de expressão praticada desde cedo. Existem relatos de que na Antiguidade os povos primitivos a utilizavam em situações relevantes do cotidiano como, por exemplo, o nascimento, a morte, o clamar ao sol e a chuva, a plantação e a colheita, a pesca e a caça, dentre outras. 3

$\mathrm{Na}$ atualidade, pode ser utilizada como uma prática de exercício físico e, principalmente, dentro do ambiente de academias de ginástica e musculação, vem sendo considerada uma das maneiras mais simples de exercitar o corpo e a mente. 4 Com isso, é notável o crescimento das aulas de dança, correspondente também a busca pela modalidade entre os públicos de diferentes faixas etárias. Essa ascensão da prática regular em academias ocorreu, principalmente, devido ao papel agregador que a dança acarreta o aperfeiçoamento dos âmbitos físico, psíquico, nas relações interpessoais e no lazer. 5

Nesta perspectiva, a dança, praticada de forma regular, integra sentidos mais vastos do que aprender uma coreografia, ou memorizar e exercer uma série de movimentos. Envolve, sobretudo, diversos benefícios, tais como a melhora da resistência muscular localizada e da coordenação motora, além de contribuir positivamente para aumentos da flexibilidade, agilidade e equilíbrio.5,6 A literatura ainda aponta que a dança estimula a comunicação, eleva a autoestima,7,8 além de contribuir para manutenção da massa óssea, reduzindo os efeitos deletérios do envelhecimento. 9

De fato, alguns estudos prévios, realizados em ambiente laboratorial, comprovam que intervenções com a prática da dança cronicamente melhora a qualidade de vida de idosos. ${ }^{10,11,12}$ Mesmo com os benefícios que esta atividade promove em diversos aspectos relacionados à saúde e ao condicionamento físico,7,9 constata-se que poucos estudos na literatura investigam a qualidade de vida de praticantes de dança em academias. Desta forma, é necessária essa investigação, visto que, a pandemia, relacionada ao novo Coronavírus (SARSCOV-2) $)^{13,14}$ causador da COVID-19, vem impactando negativamente a saúde da população e a economia mundial. ${ }^{15}$

Além disso, devido à falta de profilaxia, tratamentos específicos e sua alta taxa de transmissibilidade, o Brasil adotou o isolamento social como a principal medida de prevenção e controle da doença da COVID-19. ${ }^{16}$ Com isso, foi suspensa grande parte das atividades laborais, físicas e recreativas, sendo proibida a permanência em praias e praças públicas. ${ }^{17}$ Mesmo constituindo-se em uma medida estratégica para a contenção de casos, o isolamento social pode acarretar consequências negativas, tais como, o aumento do sedentarismo e inatividade física da população. ${ }^{18}$

Ademais, este isolamento prolongado, limitado a residência, pode diminuir a interação social e alterar o nível motivacional para a prática de atividades físicas, pois estas, muitas vezes, são associadas a necessidade de estrutura física, equipamentos complexos e de alto custo. ${ }^{17}$ Diante disto, o objetivo do presente estudo é analisar a percepção da qualidade de vida de praticantes de dança em academias na cidade de João Pessoa-PB no período pandêmico da COVID-19. 


\section{MATERIAL E MÉTODOS}

\section{Tipo da Pesquisa}

O presente estudo caracterizou-se como uma pesquisa de campo, descritiva e com abordagem quantitativa, que avaliou a qualidade de vida de praticantes de danças em academias na cidade de João Pessoa-PB. Este estudo ocorreu no período pandêmico da COVID-19, no qual a cidade se encontrava classificada como bandeira amarela. Nessa situação, podiam funcionar serviços essenciais, cumprindo novos protocolos de segurança sanitária, porém, a população estava com a mobilidade reduzida.

\section{Participantes}

A amostra foi composta por 101 pessoas, entre homens e mulheres (30,2 \pm 10,9 anos de idade; tempo de prática $2,7 \pm 2,6$ anos), praticantes de dança em academias. Os critérios de inclusão do presente estudo foram: a) estar frequentando aulas de dança em academias; b) ter idade mínima de 18 anos; c) ser praticante de dança há pelo menos dois meses. Não foram excluídos sujeitos no presente estudo.

A pesquisa em questão foi aprovada pelo Comitê de Ética em Pesquisa envolvendo seres humanos da Faculdade de Enfermagem Nova Esperança, com número CAAE 37129720.0.0000.5179. Foram explicados os possíveis riscos e benefícios inerentes ao presente estudo aos sujeitos e, logo após, assinaram um termo de consentimento livre esclarecido, elaborado de acordo com o Conselho Nacional de Saúde 446/12.

\section{Instrumentos de Coleta de Dados}

A percepção da qualidade de vida dos participantes foi verificada por meio do questionário WHOQOL-bref. Este instrumento se divide em quatro partes, contemplando os domínios físico, psicológico, meio ambiente e relações pessoais. As respostas dos participantes foram registradas de acordo com a escala Likert, de 1 a 5 pontos. ${ }^{19}$

O domínio físico é composto por questões que envolvem a percepção de dores e desconfortos, vigor e fadiga, qualidade de sono e repouso, entre outros; O psicológico é elaborado com questões que estão estabelecidas para a percepção dos sentimentos negativos e positivos, engloba questões relacionadas ao pensar, aprender, à memória, entre outros; $O$ domínio relações sociais é constituído por questões que compreendem a percepção acerca das relações pessoais e a interação social, do suporte, ou seja, o apoio que o indivíduo recebe no seu meio social e a atividade sexual; $O$ do meio ambiente é representado por questões que envolvem as percepções voltadas à segurança física e à proteção, recursos financeiros, sociais, cuidados de saúde, a disponibilidade e qualidade, entre outros. ${ }^{19}$

\section{Procedimentos de Coleta de Dados}

Realizou-se um primeiro contato com os proprietários das academias a fim de se obter a liberação para se realizar o presente estudo, no período de agosto de 2020, quando ocorreu a liberação e abertura das academias de musculação da cidade de João Pessoa-PB.

Logo após, foi realizado um levantamento do número de praticantes, matriculados nas aulas de dança. Foi agendada uma reunião com os professores de dança das academias para esclarecimentos sobre a pesquisa. Durante as visitas às academias, os participantes da pesquisa 
receberam esclarecimentos sobre o correto preenchimento dos questionários, a explicação do objetivo do estudo e da assinatura do termo de consentimento livre e esclarecido.

\section{Análise Estatística}

Os dados foram analisados no pacote estatístico computadorizado Statistical Package for the social Science (SPSS), versão 21.0. Utilizou-se o teste de distribuição de frequência absoluta e relativa para descrição de dados sociodemográficos e dos domínios de qualidade de vida. Além disso, utilizaramse média e desvio padrão para descrever os escores dos domínios da qualidade de vida dos praticantes de dança.

\section{RESULTADOS E DISCUSSÃO}

Em relação ao sexo dos praticantes de danças em academias, que participaram do presente estudo, verificou-se que a maioria dos avaliados são mulheres (85,1\%). Desta forma, este achado parece estar relacionado à cultura da dança, uma vez que parte da sociedade compreende que a dança é uma modalidade voltada ao público feminino. ${ }^{20}$ No entanto, baseando-se no que discutem os pensamentos advindos do contexto histórico, cultiva-se uma concepção preconceituosa envolvendo o homem e a dança, fazendo, assim, com que ocorra um distanciamento do público masculino nessas aulas. ${ }^{21}$

Em relação aos domínios do questionário de qualidade de vida, observouse que a maioria dos avaliados se classificou como regular nos domínios físico $(81,2 \%)$. psicológico $(66,3 \%)$ e meio ambiente $(64,4 \%)$. No entanto, constatou-se que a maioria apresenta-se na classificação boa no domínio relação social $(51,2 \%)$ (Tabela 1$)$.

TABELA 1: Frequência absoluta (n) e relativa (\%) da classificação nos diferentes domínios da qualidade de vida de praticantes de dança em academias de João Pessoa-PB, no período pandêmico da COVID-19 $(n=101)$

\begin{tabular}{|c|c|c|}
\hline \multirow{2}{*}{ VARIÁVEL } & \multicolumn{2}{|c|}{ FREQUÊNCIA } \\
\hline & $\mathbf{n}$ & $\%$ \\
\hline \multicolumn{3}{|l|}{ Domínio Físico } \\
\hline Necessita melhorar & 15 & 14,9 \\
\hline Regular & 82 & 81,2 \\
\hline Boa & 4 & 4,0 \\
\hline Muito boa & 0 & 0 \\
\hline \multicolumn{3}{|l|}{ Domínio Psicológico } \\
\hline Necessita melhorar & 2 & 5,0 \\
\hline Regular & 67 & 66,3 \\
\hline Boa & 29 & 28,7 \\
\hline Muito boa & 0 & \\
\hline \multicolumn{3}{|c|}{ Domínio Relação Social } \\
\hline Necessita melhorar & 2 & 2,0 \\
\hline Regular & 3 & 38,6 \\
\hline Boa & 52 & 51,5 \\
\hline Muito boa & 8 & 7,9 \\
\hline \multicolumn{3}{|c|}{ Domínio Meio Ambiente } \\
\hline Necessita melhorar & 2 & 2,0 \\
\hline Regular & 65 & 64,4 \\
\hline Boa & 34 & 33,7 \\
\hline Muito boa & 0 & 0 \\
\hline
\end{tabular}


Na análise da média dos domínios que representam a qualidade de vida de indivíduos, constatou-se que o domínio relação social
$(15,6 \pm 0,5)$ apresentou um maior escore para os praticantes de dança em academias.

TABELA 2: Análise descritiva do nível de qualidade de vida de praticantes de dança em academias de João Pessoa-PB, no período pandêmico da COVID-19 $(n=101)$

\begin{tabular}{lc}
\hline \hline Domínios de QV (WHOQOL Bref) & X \pm dp \\
\hline \hline Domínio 1- Físico & $13,4 \pm 1,5$ \\
Domínio 2- Psicológico & $14,6 \pm 1,6$ \\
Domínio 3- Relações Sociais & $15,6 \pm 2,6$ \\
Domínio 4- Meio Ambiente & $14,6 \pm 1,8$ \\
\hline \hline & \multicolumn{2}{c}{ Legenda: QV = Qualidade de Vida; X = média; } \\
& dp = desvio padrão
\end{tabular}

O nível de qualidade de vida dos praticantes de dança em academias é satisfatório, porém, constatou-se que - domínio relação social apresenta um percentual $(77,2 \%)$ maior que os domínios físico, psicológico e meio ambiente. Diante dos dados analisados, apesar de os praticantes de dança apresentarem a qualidade de vida satisfatória, nota-se que apenas o domínio relações sociais está mais próximo do 100\%, que é o valor considerado o ápice da qualidade de vida, e isto implica dizer que os domínios físico, psicológico e meio ambiente podem ter sido influenciados pelo momento atual em que estamos vivendo, a pandemia COVID-19..$^{18,22}$ O impacto que a pandemia ocasionou para a população pode ser considerado como motivo para que o nível de satisfação física, psíquica e do meio ambiente se encontre na classificação regular, tendo em vista que, a atual circunstância de isolamento social e números crescentes de óbitos, acabem acarretando limitações nas atividades do cotidiano. ${ }^{13,14}$

O isolamento social também pode implicar na redução das atividades físicas, fazendo com que o indivíduo passe por longo tempo de comportamento sedentário, provocando um processo de regressão funcional, problemas articulares e posturais. ${ }^{14,17}$ Tal processo age sobre o psicológico, como o estresse e a ansiedade, desencadeando ou agravando doenças de origem psicológica. ${ }^{18}$ Além disso, a pandemia de COVID-19 tem ocasionado impactos econômicos negativos, assim como o aumento da ocupação das unidades hospitalares. Portanto, os fatos mencionados justificam a classificação regular nos domínios físico, psicológico e meio ambiente.

Outro aspecto importante nesses resultados é de que o baixo valor no domínio ambiente pode ter ocorrido devido a influência de algumas facetas que formam este domínio, tais como, segurança física, proteção e ambiente do lar que apresentaram um percentual baixo. Com isso, acredita-se que com o nível de violência aumentando nas cidades de todo o país, a população sofre o impacto negativo que indiretamente afeta aspectos emocionais, como estresse, ansiedade e com o isolamento social as ruas com redução de pessoas pode provocar medo de sair de suas casas para realização de tarefas diárias. $^{23}$

O estudo apresenta algumas limitações, dentre essas, o fato de não ter analisado o nível de aptidão física dos participantes, o que poderia explicar melhor os resultados. Além disso, não foi utilizada uma escala psicométrica que avaliasse o estado de humor dos praticantes de aulas de dança para constatar o perfil psicológico dos integrantes da pesquisa. 


\section{CONCLUSÃO}

As aulas de dança em academias são compostas, em sua grande maioria pelo público feminino e os praticantes de dança apresentaram escores positivos para os domínios da qualidade de vida. Sugere-se que futuros estudos realizem associações entre o questionário WHOQOL-Bref e algumas escalas psicométricas, tais como o questionário

\section{REFERÊNCIAS BIBLIOGRÁFICAS}

1. Rocha MD. Dança de salão instrumento para a qualidade de vida. Movimento \& Percepção. 2007; 7(10): 76-89.

2. Duarte JC. Fundamentos estéticos da educação. Campinas, SP: Papirus, 1995.

3. Barancelli LLR, Pawlowystsch PWM. Dança e qualidade de vida: um estudo biopsicossocial. Repert. 2016; 19(26): 273-82.

4. Costa VF, Moura SKMSF, Lopes DT. Estudo da demanda pela prática de dança aeróbica em academias. Revista Campo do saber. 2018; 4(1): 120-36.

5. Machado Z, Santos GR, Guimarães ACA, Fernandes S, Soares A. Qualidade de vida dos praticantes de dança de salão. Revista Brasileira de Atividade Física e saúde. 2012; 17(1):39-45.

6Yan AF, Cobley S, Chan C, Pappas E, Nicholson LL, Ward RE, et al.. The Effectiveness of Dance Interventions on Physical Health Outcomes Compared to Other Forms of Physical Activity: A Systematic Review and Meta-Analysis. Sports Med. 2018; 48(4): 933-51.

7. Souza JCL, Metzner AC. Benefícios da dança no aspecto social e físico dos idosos. Revista internacional de atividade física, escala de Brunel, para explicar melhor os efeitos da pandemia nesta população. Ademais, poderia ser feito um levantamento dos dados analisados e realizada uma comparação entre os níveis de qualidade de vida do sexo feminino e masculino.

Fafibe On-line. 2013; 6(6): 8-13.

8. Nanni D. Dança educação: princípios, métodos e técnicas. Rio de Janeiro: Sprint, 2001.

9. Souza MF, Marques JP, Vieira AF, Santos DC, Cruz AE. Contribuições da dança para a qualidade de vida de mulheres idosas. EF Deportes.com, Revista Digital. 2010; 15(148): 1-5.

10. Gouvêa JAG, Antunes MD, Bortolozzi F, Marques AG, Bertolini SMMG. Impact of senior dance on emotional and motor parameters and quality of life of the elderly. Rev Rene. 2017; 18(1): 51.

11. Castro JC, Bastos FAC, Cruz THP, Giani TS, Ferreira MA, Dantas EHM. Níveis de qualidade de vida em idosas ativas praticantes de dança, musculação e meditação. Rev Bras Geriatr Gerontol. 2009; 12(2): 255-65.

12. Oliveira LC, Pivoto EA, Vianna PCP. Análise dos resultados de qualidade de vida em idosos praticantes de dança sênior através do SF-36. Acta Fisiatr. 2009; 16(3): 101-04.

13. Ksiazek TG, Erdman D, Goldsmith CS, Zaki SR, Peret T, Emery S, et al.; SARS Working Group. A novel coronavirus associated with severe acute 
respiratory syndrome. N Engl J Med. 2003; 348: 1953-66.

14. Li Q, Guan X, Wu P, Wang X, Zhou L, Tong Y, et al. Early Transmission Dynamics in Wuhan, China, of Novel Coronavirus-Infected Pneumonia. N Engl J Med. 2020; 382:1199-207.

15. Ayittey FK, Ayittey MK, Chiwero NB, Kamasah JS, Dzuvor C. Economic impacts of Wuhan 2019nCoV on China and the world. J Med Virol 2020; 92: 473-75.

16. Anderson RM, Heesterbeek H, Klinkenberg D, Hollingsworth TD. How will country-based mitigation measures influence the course of the COVID-19 epidemic? Lancet 2020; 395:931-34.

17. Filho BABS, Tritany EF. COVID-19: importância das novas tecnologias para a prática de atividades físicas como estratégia de saúde pública. Cad. Saúde Pública 2020; 36(5): 1-5.

18. Liu CH, Stevens C, Conrad RC, Hahm HC. Evidence for elevated psychiatric distress, poor sleep, and quality of life concerns during the COVID-19 pandemic among U.S. young adults with suspected and reported psychiatric diagnoses. Psychiatry Research. 2020; 292: 113345.

19. Fleck MPA, Lousada S, Xavier M, Vieira ECG, Santos L, Pinzon V. Aplicação da versão em português do instrumento abreviado de avaliação da qualidade de vida "WHOQOL-bref" Rev. Saúde Públ. 2001; 34(2): 178-83.

20. Nelza DSP, Verônica V. O preconceito e o homem que dança: Uma reflexão nas aulas de educação física. Cadernos PDE. 2013; 1(1): 1-11.

21. Santos LB, Filho RAF. Dança versus masculinidade: o ‘homem' do século XXI e as nuances sociais relacionadas à discriminação. Revista Lecturas Educación y Esportes. 2012; 17(168): 1-5.

22. Barbosa BT, Lima-Junior D, Filho EMS. The impact of COVID-19 on sporting events and highperformance athletes. Sports Med Phys Fitness. 2020; 60(11): 1507-8.

23. Koury MGP. Medos urbanos e mídia: o imaginário sobre juventude e violência no Brasil atual. Soc. Estado. 2011; 26(3): 471-86. 